\title{
Matching Quadrupoles for AGS Helical Snake:
}

\author{
E. D. Courant \\ Brookhaven National Laboratory
}

Assume: a helical snake inserted in AGS section I-20 with Parameters:

Total length $2.1 \mathrm{~m}$, field strength 3.0 Tesla.

First section: $44.5 \mathrm{~cm}$, twist 180 degrees

Second section: $1.21 \mathrm{~m}$, twist 245 degrees

Third section: $44.5 \mathrm{~cm}$, twist 180 degrees.

Assume (for the sake of manageable analytic computation): abrupt transitions from zero field to full field at ends of helix; abrupt change of pitch between end and center sections.

At injection energy (Ggamma $=4.7$ ) the snake strength is $34.9 \%$, and the trajectory is as shown

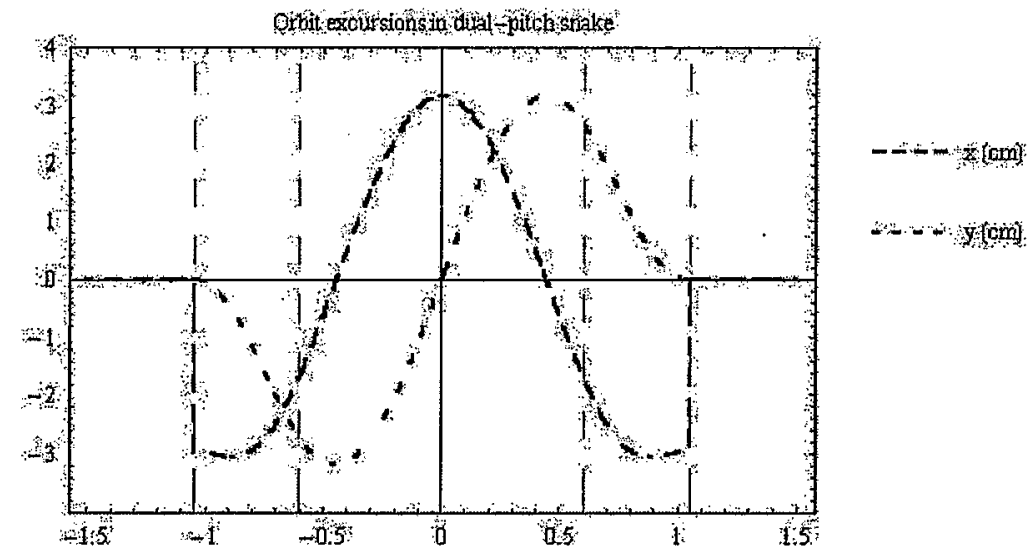

Because of Maxwell's equations, a helical field automatically include nonlinearities, local field gradients and solenoidal fields, varying over the trajectory. Averaging these over the trajectory, we can compute the effective transfer matrix for the helix, which unfortunately is nothing like that for a drift space of the same length. For the whole 10foot $(3.048 \mathrm{~m})$ straight section including the snake, this matrix comes out to be

*Work performed under the auspices of the US Department of Energy Sources and Polarimeters, edited by Y. I. Makdisi, A. U. Luccio, and W. W. MacKay 우 2003 American Institute of Physics 0-7354-0136-5/03/\$20.00 


$\begin{array}{rrrr}0.664572 & 2.646738 & -0.03787 & -0.1274 \\ -0.21013 & 0.664572 & 0.007435 & -0.03248 \\ 0.032485 & 0.127396 & 0.845606 & 2.809608 \\ -0.00743 & 0.037874 & -0.10064 & 0.845606\end{array}$

which includes significant coupling terms (upper right and lower left corners). The fields on the trajectory (again calculated with the hard-edge approximations) are

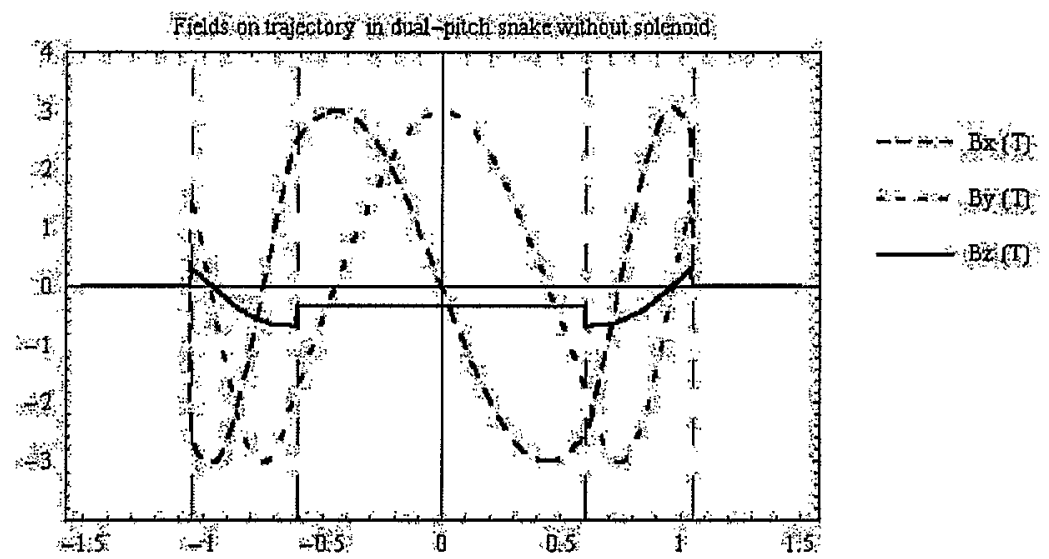

By superimposing a suitable solenoidal field we can eliminate the coupling terms. With a solenoidal field of $0.3371 \mathrm{~T}$ the fields are as shown below:

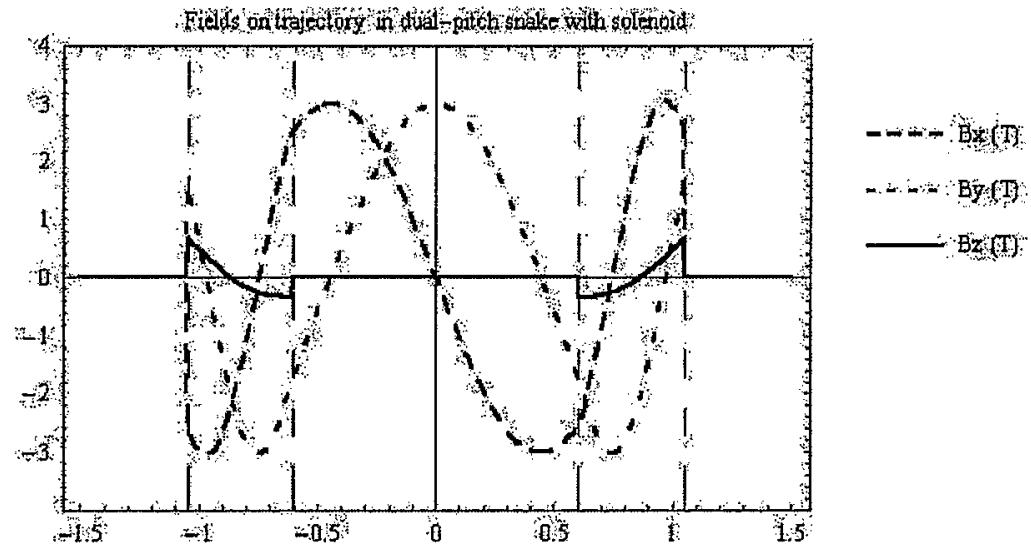

the snake is now $27.7 \%$ of a full snake, and the matrix is altered to 


$\begin{array}{rrrr}0.666718 & 2.651499 & -7.21 \mathrm{E}-06 & -2.9 \mathrm{E}-05 \\ -0.2095 & 0.666718 & 1.59 \mathrm{E}-06 & -9.05 \mathrm{E}-06 \\ 9.05 \mathrm{E}-06 & 2.92 \mathrm{E}-05 & 0.848034 & 2.814629 \\ -1.59 \mathrm{E}-06 & 7.21 \mathrm{E}-06 & -0.09978 & 0.848034\end{array}$

i.e. the coupling is eliminated. But the matrix is a long way from a simple drift matrix; this causes a huge mismatch of the orbit stability. With the tunes of the plain AGS set at $(8.1,8.75)$ the tunes come out as $(8.29,8.88)$, i.e. a tune change of around 0.2 units, and the orbit functions are as shown:

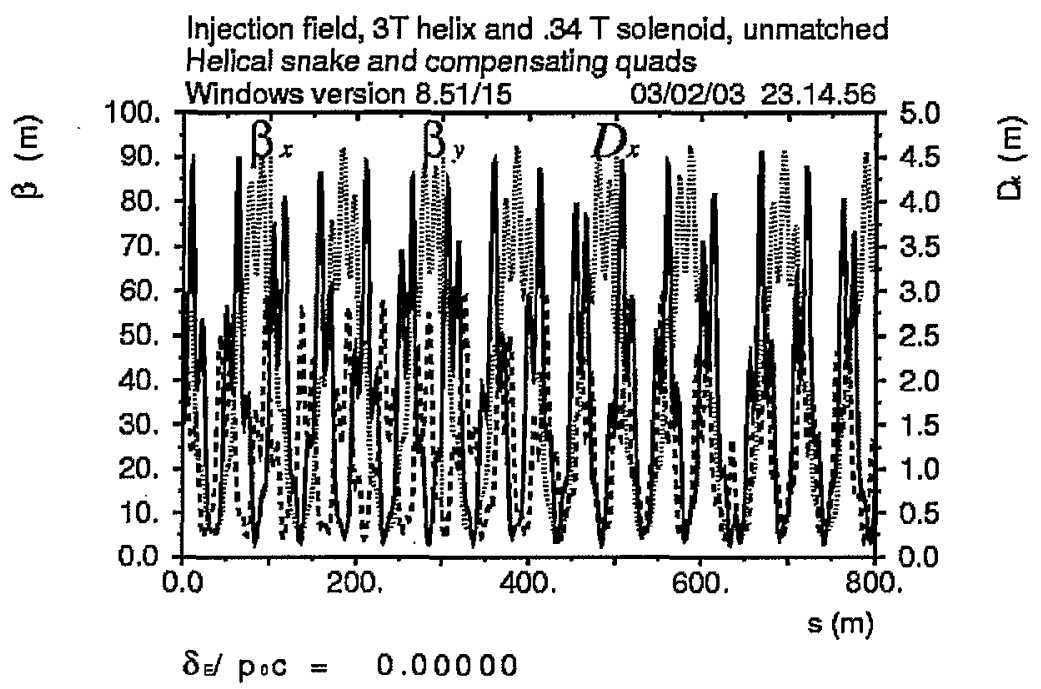

(Here the snake is located in the last superperiod, i.e. at $\mathrm{s}=790 \mathrm{~m}$ ). Recall that in the bare AGS the maximum beta functions are about $22 \mathrm{~m}$, and the dispersion function 1.8 m.)

Using the MAD program we find that with quadrupoles in sections $\mathrm{I17}, \mathrm{I19}, \mathrm{J} 1, \mathrm{~J} 3$ (the sections nearest to the snake at 120 ) we can match the beta functions perfectly (except, of course, in the section containing the snake).

The quadrupoles are assumed to have effective length of $35 \mathrm{~cm}$, and their strengths are calculated as

$\mathrm{I} 17=1.82 \mathrm{~T} / \mathrm{m}$,

$I 19=1.63 \mathrm{~T} / \mathrm{m}$,

$\mathrm{J} 1=-0.92 \mathrm{~T} / \mathrm{m}$,

$\mathrm{J} 3=1.92 \mathrm{~T} / \mathrm{m}$. 
These are quite modest, and should be easy to put in. With these quadrupoles the orbit functions become:

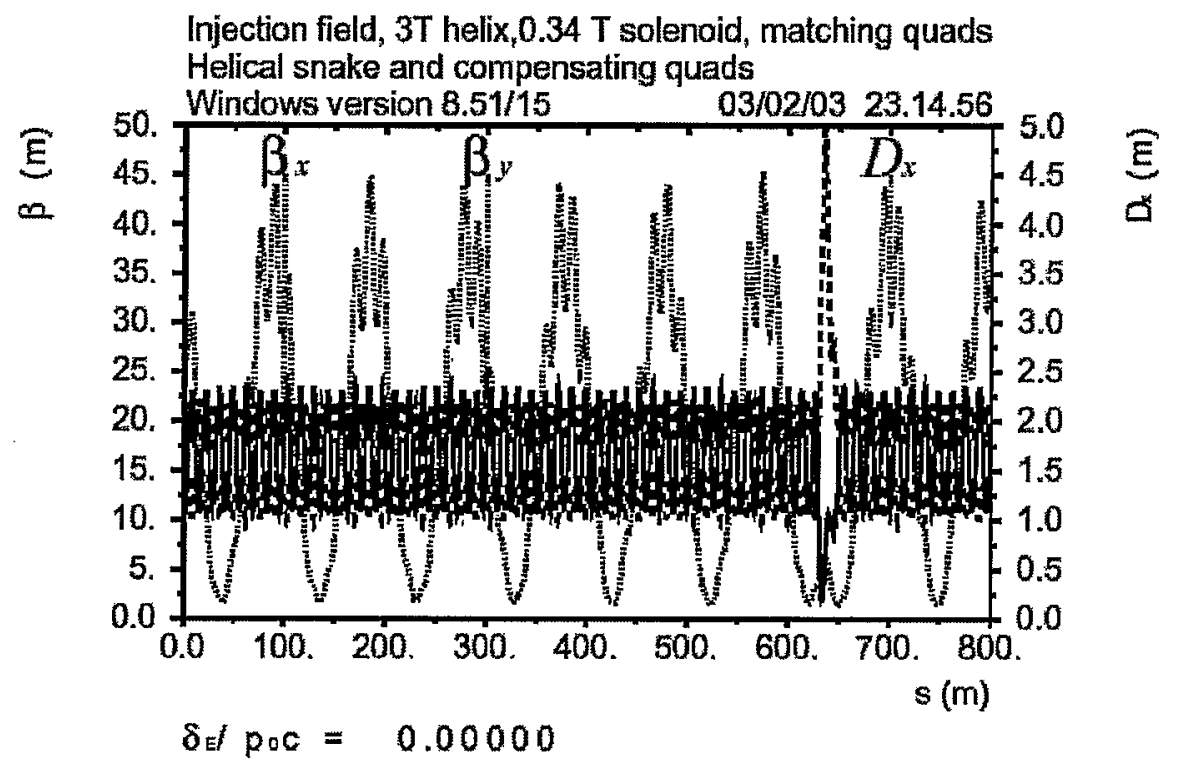

Note that the beta functions are perfectly matched, but the dispersion function is not (no attempt was made to match it - undoubtedly that could be done too). The tunes are 8.65 and 8.97; this could easily be adjusted.

At higher energies the effect of the snake on the orbit is weaker. At Ggamma $=8.9$ (the $0+\mathrm{nu}$ resonance) the unmatched beta functions have a maximum of 39 meters as against 90 at injection, and the solenoid needed to counteract coupling is down to 0.17 Tesla. The compensating quadrupoles are, for one set of tunes,

$\mathrm{I17}=3.46 \mathrm{~T} / \mathrm{m}$,

$\mathrm{I} 19=-0.65 \mathrm{~T} / \mathrm{m}$,

$\mathrm{J} 1=2.12 \mathrm{~T} / \mathrm{m}$,

$\mathrm{J} 3=0.65 \mathrm{~T} / \mathrm{m}$.

Finally, at high energy no compensation is needed. In all cases the tunes in my examples are about 8.65 and 8.95 . Another cost of the compensating quads is that they break the 12-fold periodicity of the AGS lattice; therefore in addition to the intrinsic resonances at $G \gamma=12 k \pm v$ there are now resonances at

$$
G \gamma=k \pm v
$$

with all integers $k$. Thus with the corrections at injection energy the spectrum of resonances is now 


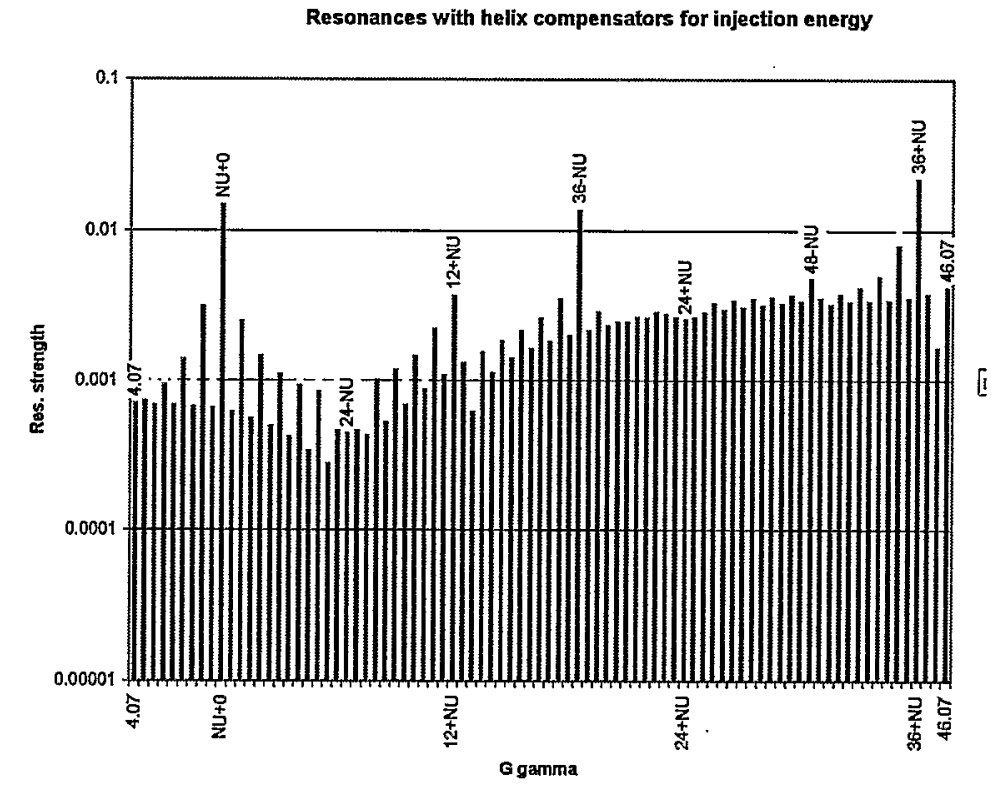

Thus new resonances have now appeared. But the ones near injection energy are weak enough so that, with the partial snake, they will not cause any trouble.

At higher energies the compensating quadrupoles are relatively weaker, and the resonances they cause are correspondingly weaker. With the compensating quadrupoles for $G \gamma=8.9$ the resonance spectrum is

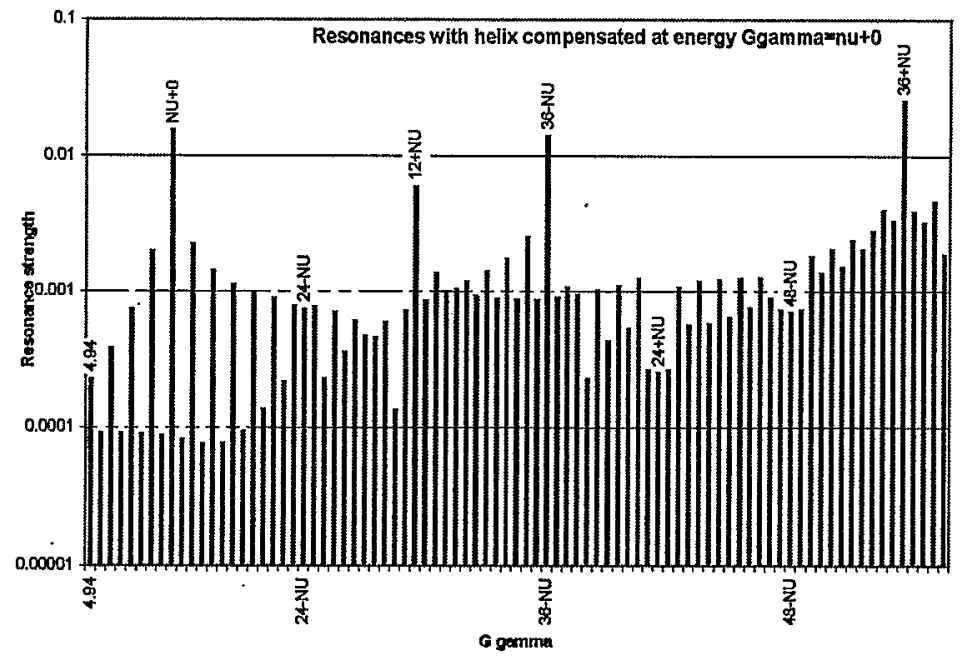

and the symmetry-breaking resonances are weak compared to the main $0+v$ resonance, so clearly the will not affect the partial snake's ability to cope with resonances. 